\title{
Excretion Rate From T1 to T2 Normalized by WT
}

National Cancer Institute

\section{Source}

National Cancer Institute. Excretion Rate From T1 to T2 Normalized by WT. NCI

Thesaurus. Code C105453.

The excretion rate over the interval from $\mathrm{T} 1$ to $\mathrm{T} 2$ divided by the weight. 